\title{
New dual gravitational charges
}

\author{
Hadi Godazgar, ${ }^{1, *}$ Mahdi Godazgar, ${ }^{2, \dagger}$ and C. N. Pope ${ }^{3,4, \$}$ \\ ${ }^{1}$ Max-Planck-Institut für Gravitationsphysik (Albert-Einstein-Institut), \\ Mühlenberg 1, D-14476 Potsdam, Germany \\ ${ }^{2}$ Institut für Theoretische Physik, Eidgenössische Technische Hochschule Zürich, \\ Wolfgang-Pauli-Strasse 27, 8093 Zürich, Switzerland \\ ${ }^{3}$ George P. \& Cynthia Woods Mitchell Institute for Fundamental Physics and Astronomy, \\ Texas A\&M University, College Station, Texas 77843, USA \\ ${ }^{4}$ DAMTP, Centre for Mathematical Sciences, Cambridge University, \\ Wilberforce Road, Cambridge CB3 OWA, United Kingdom
}

(Received 14 December 2018; published 7 January 2019)

\begin{abstract}
We show that there are a further infinite number of, previously unknown, supertranslation charges. These can be viewed as duals of the known Bondi-Metzner-Sachs (BMS) charges corresponding to supertranslations. In Newman-Penrose language, these new supertranslation charges roughly correspond to the imaginary part of the leading term in $\psi_{2}$. We find these charges by dualizing the Barnich-Brandt asymptotic charges and argue that this prescription gives rise to new bona fide charges at null infinity.
\end{abstract}

DOI: 10.1103/PhysRevD.99.024013

\section{INTRODUCTION}

Recently, the relation between Bondi-Metzner-Sachs (BMS) symmetry and Newman-Penrose charges at null infinity of asymptotically flat spacetime has been made explicit in linear and nonlinear gravity [1,2], as well as electromagnetism [3,4]. While BMS charges are strictly defined at null infinity, and in particular include the Bondi 4-momentum, it has been shown that other charges can be defined by extending the definition of BMS charges into the bulk and it is these extended BMS charges that encompass some of the Newman-Penrose charges. In linearized gravity, at each order in a $1 / r$ expansion away from null infinity the Newman-Penrose charges are components of the Weyl scalar $\psi_{0}$ in a $1 / r$ expansion [5] - the real parts of which correspondingly extend the notion of BMS charges as a $1 / r$ expansion into the bulk [1]. Furthermore, the same picture holds in the nonlinear theory, where an extension of the BMS charges using the BarnichBrandt prescription [6] as a $1 / r$ expansion away from null infinity is shown to include 5 of the 10 nonlinear NewmanPenrose charges [2]. It remains an open question whether the extension of the BMS charges into the bulk can be

\footnotetext{
*hadi.godazgar@aei.mpg.de †m.godazgar@qmul.ac.uk pope@physics.tamu.edu
}

Published by the American Physical Society under the terms of the Creative Commons Attribution 4.0 International license. Further distribution of this work must maintain attribution to the author(s) and the published article's title, journal citation, and DOI. Funded by SCOAP ${ }^{3}$. further enlarged such that they contain the imaginary parts of the Newman-Penrose charges. In this paper we will not resolve this question in the general setting of extended BMS charges but show that already at the level of the standard BMS charges something has been hitherto missed.

At leading order, the BMS charges can be derived from the Barnich-Brandt formalism [7]. By making a particular choice of the supertranslation parameter $s(\theta, \phi)$, namely choosing $l=0,1$ spherical harmonics, ${ }^{1}$ the BMS charge can be shown to include the real part ${ }^{2}$ of the Bondi 4-momentum [7]

$$
P_{\ell, m}=-\frac{1}{2 \sqrt{\pi} G} \int d \Omega Y_{\ell m}\left(\psi_{2}^{0}+\sigma^{0} \partial_{u} \bar{\sigma}^{0}\right)
$$

where $\ell=0$ or 1 , and $\psi_{2}^{0}$ and $\sigma^{0}$ are the leading terms in a $1 / r$-expansion of the Weyl scalar $\psi_{2}$ and the shear $\sigma$, respectively:

\footnotetext{
${ }^{1}$ The supertranslation parameter describing a diffeomorphism of a physical metric should, of course, be real. It is convenient to decompose a general such parameter $s(\theta, \varphi)$ as a sum over spherical harmonics, which we may think of as the complete set of (real) solutions of $\square s=-\ell(\ell+1) s$ on the unit sphere, where $\ell=0,1,2, \ldots$. It will always be understood that we are taking $s(\theta, \phi)$ to be real. Of course in practice it is often convenient to work with the complex basis of spherical harmonics $Y_{\ell m}(\theta, \phi)$. Whenever, in this paper, we speak of taking $s(\theta, \phi)$ to be a harmonic $Y_{\ell m}(\theta, \phi)$, it should be understood that really, we mean that $s$ is a real function constructed as an appropriate linear combination of the complex $Y_{\ell m}(\theta, \phi)$ harmonics.

${ }^{2}$ To be precise, the real part of $-1 /(4 G) \int d \Omega s\left(\psi_{2}^{0}+\sigma^{0} \partial_{u} \bar{\sigma}^{0}\right)$, where $s$ is any of the four linearly independent real harmonics proportional to $Y_{0,0}, Y_{1,0},\left(Y_{1,1}-Y_{1,-1}\right)$ or $i\left(Y_{1,1}+Y_{1,-1}\right)$.
} 


$$
\psi_{2}^{0}=\lim _{r \rightarrow \infty} r^{3} \psi_{2}, \quad \text { and } \quad \sigma^{0}=\lim _{r \rightarrow \infty} r^{2} \sigma .
$$

For $\ell=0$ or 1 , the fact that the Barnich-Brandt prescription gives only the real part is not so troubling, since one can show that

$$
\Im\left(\psi_{2}^{0}+\sigma^{0} \partial_{u} \bar{\sigma}^{0}\right)=\Im\left(\bar{\partial}^{2} \sigma^{0}\right) .
$$

Now, $\bar{\partial}^{2} Y_{\ell m}=0$ for $\ell=0$ or 1 , and so the imaginary part is a total derivative, which vanishes under the integral over the sphere.

If we consider instead an arbitrary supertranslation parameter, then

$$
s(\theta, \phi) \Im\left(\psi_{2}^{0}+\sigma^{0} \partial_{u} \bar{\sigma}^{0}\right)=s(\theta, \phi) \Im\left(\bar{ð}^{2} \sigma^{0}\right)
$$

is no longer a total derivative when $\ell \geq 2$. Thus, one may ask if there is a sense in which the Barnich-Brandt prescription is only giving half of the asymptotic charges when $\ell \geq 2$ [i.e., only the real part of the complex generalized charge $\left.-1 /(4 \pi G) \int d \Omega s\left(\psi_{2}^{0}+\sigma^{0} \partial_{u} \bar{\sigma}^{0}\right)\right]$. It is this question that we shall address in this paper. Indeed, as we shall show, we may define an infinite number of extra supertranslation charges. These charges are obtained by considering the "dual" of the Barnich-Brandt asymptotic charge, which is the analog of considering the field strength and its dual in the case of electromagnetism $[3,4]$. In a gravitational context, it is analogous to getting a NUT charge by dualizing the Bondi mass [8] or Komar mass [9].

In Sec. II, we consider for illustrative purposes the simpler case of electromagnetism and show how the usual electric and magnetic charges can be viewed as the real and imaginary parts of the Newman-Penrose charge, respectively. We extend this analogy to the gravitational case in Sec. III and find that one can define dual gravitational charges corresponding to the supertranslation generators of the BMS group at null infinity. We conclude with some comments in Sec. IV.

\section{ELECTROMAGNETISM}

We begin by considering the simpler case of electromagnetism on flat Minkowski spacetime [3,4], with metric given in outgoing Eddington-Finkelstein coordinates $\left(u, r, x^{I}=\{\theta, \phi\}\right)$ by

$$
d s^{2}=-d u^{2}-2 d u d r+r^{2} \omega_{I J} d x^{I} d x^{J}
$$

A convenient choice of complex null frame $e_{\mu}{ }^{a}=$ $\left(\ell^{a}, n^{a}, m^{a}, \bar{m}^{a}\right)$ is given by

$$
\begin{aligned}
\ell & =\frac{\partial}{\partial r}, \quad n=\frac{\partial}{\partial u}-\frac{1}{2} \frac{\partial}{\partial r}, \quad m=\frac{\hat{m}^{I}}{r} \frac{\partial}{\partial x^{l}}, \\
\ell^{b} & =-d u, \quad n^{b}=-\left(d r+\frac{1}{2} d u\right), \quad m^{\mathrm{b}}=r \hat{m}_{I} d x^{I}, \\
\hat{m} & =\frac{1}{\sqrt{2}}\left(\frac{\partial}{\partial \theta}+\frac{i}{\sin \theta} \frac{\partial}{\partial \phi}\right), \quad \hat{m}^{b}=\frac{1}{\sqrt{2}}(d \theta+i \sin \theta d \phi) .
\end{aligned}
$$

Following Barnich and Brandt [6], we define the electric charge to $\mathrm{be}^{3}$

$$
\mathcal{Q}_{c}=\frac{1}{4 \pi} \int_{S} c \star F=\frac{1}{4 \pi} \int_{S} d \Omega c r^{2} F_{01},
$$

where $c(x)$ is an arbitrary function on the 2-sphere corresponding to the asymptotic symmetry for electromagnetism and we use the notation that for some arbitrary covector $V$

$$
\begin{aligned}
\ell^{a} V_{a} & \equiv V_{0}=-V^{1}, \quad n^{a} V_{a} \equiv V_{1}=-V^{0}, \\
m^{a} V_{a} & \equiv V_{m}=V^{\bar{m}} .
\end{aligned}
$$

Contrast the above expression with the Newman-Penrose charge [5], generalized to include a constant $c$

$$
\mathcal{Q}_{c}^{(N P)}=\lim _{r \rightarrow \infty} \frac{1}{2 \pi} \int_{S} c r^{2} \Phi_{1},
$$

where

$$
\Phi_{1}=\frac{1}{2}\left(F_{01}+F_{m \bar{m}}\right)
$$

is a Newman-Penrose scalar corresponding to a particular component of the Maxwell field strength in the complex null frame. We only take the leading Newman-Penrose charge and do not, here, consider a $1 / r$-expansion in which case one could define a charge at every order. We stress that what appears in integral (2.5) is the complex NewmanPenrose scalar $\Phi_{1}$ multiplied by a constant. Note that the real part of $\Phi_{1}$ is given by $F_{01}$, which corresponds to the expression in the Barnich-Brandt integral (2.3). What about the imaginary part of the generalized Newman-Penrose charge given by $F_{m \bar{m}}$ ?

As emphasized above, the Barnich-Brandt integral with $c=1$ corresponds to the electric charge. Correspondingly, the asymptotic magnetic charge may be defined as

$$
\tilde{\mathcal{Q}}_{c}=\frac{1}{4 \pi} \int_{S} c F=\frac{1}{4 \pi} \int_{S} d \Omega i c r^{2} F_{m \bar{m}} .
$$

\footnotetext{
${ }^{3}$ Note that in the case of electromagnetism, the Barnich-Brandt charge is integrable. This is not the case in nonlinear gravity due to Bondi news (or more generally fake news [2]) at null infinity.
} 
Given this we conclude that

$$
\mathcal{Q}_{c}^{(N P)}=\mathcal{Q}_{c}-i \tilde{\mathcal{Q}}_{c},
$$

i.e., the generalized Newman-Penrose charge contains information about both the electric and magnetic charge.

Aside.-It may be argued that for $c=1, \tilde{\mathcal{Q}}=0$, as follows: Stokes's theorem implies that

$$
\tilde{\mathcal{Q}}=\frac{1}{4 \pi} \int_{S} F=\frac{1}{4 \pi} \int_{\Sigma} d F=0
$$

by the Bianchi identity. However, this result follows if null infinity is the only boundary of the spacetime. On a black hole background this result need not hold as the magnetic charge at infinity is equal and opposite to a contribution to the integral from the horizon.

\section{GRAVITY}

As is to be expected, the case of gravity is more intricate compared to the electromagnetic case. Starting from an asymptotically flat spacetime $[10,11]$, which we define to be a spacetime for which there exist Bondi coordinates $\left(u, r, x^{I}=\{\theta, \phi\}\right)$ in which the metric takes the form

$$
\begin{aligned}
d s^{2}= & -F e^{2 \beta} d u^{2}-2 e^{2 \beta} d u d r \\
& +r^{2} h_{I J}\left(d x^{I}-C^{I} d u\right)\left(d x^{J}-C^{J} d u\right)
\end{aligned}
$$

with the metric functions satisfying the following fall-off conditions at large $r$

$$
\begin{aligned}
F\left(u, r, x^{I}\right) & =1+\frac{F_{0}\left(u, x^{I}\right)}{r}+o\left(r^{-1}\right), \\
\beta\left(u, r, x^{I}\right) & =\frac{\beta_{0}\left(u, x^{I}\right)}{r^{2}}+o\left(r^{-3}\right), \\
C^{I}\left(u, r, x^{I}\right) & =\frac{C_{0}^{I}\left(u, x^{I}\right)}{r^{2}}+o\left(r^{-2}\right), \\
h_{I J}\left(u, r, x^{I}\right) & =\omega_{I J}+\frac{C_{I J}\left(u, x^{I}\right)}{r}+o\left(r^{-1}\right),
\end{aligned}
$$

where $\omega_{I J}$ is the standard metric on the round 2-sphere with coordinates $x^{I}=\{\theta, \phi\}$. Moreover, residual gauge freedom allows us to require that

$$
h=\omega,
$$

where $h \equiv \operatorname{det}\left(h_{I J}\right)$ and $\omega \equiv \operatorname{det}\left(\omega_{I J}\right)=\sin ^{2} \theta$. Furthermore, we assume that

$$
T_{0 m}=o\left(r^{-3}\right)
$$

so that the Einstein equation then implies that $[2,7]$

$$
C_{0}^{I}=-\frac{1}{2} D_{J} C^{I J}
$$

where $D_{I}$ is the covariant derivative compatible with the metric on the round 2-sphere $\omega_{I J}$.

The BMS charge is defined as [6,7]

$$
\not \mathcal{Q}=\frac{1}{8 \pi G} \lim _{r \rightarrow \infty} \int_{S} \star H=\frac{1}{8 \pi G} \lim _{r \rightarrow \infty} \int_{S} d \Omega r^{2} e^{2 \beta} H^{u r},
$$

where

$$
\begin{aligned}
H= & \frac{1}{2}\left\{\xi_{b} g^{c d} \nabla_{a} \delta g_{c d}-\xi_{b} \nabla^{c} \delta g_{a c}+\xi^{c} \nabla_{b} \delta g_{a c}\right. \\
& \left.+\frac{1}{2} g^{c d} \delta g_{c d} \nabla_{b} \xi_{a}+\frac{1}{2} \delta g_{b c}\left(\nabla_{a} \xi^{c}-\nabla^{c} \xi_{a}\right)\right\} d x^{a} \wedge d x^{b}
\end{aligned}
$$

and the notation $\not$ is used to signify the fact that the expression is not necessarily integrable. The asymptotic symmetry generator

$\xi=s \partial_{u}+\int d r \frac{e^{2 \beta}}{r^{2}} h^{I J} D_{J} s \partial_{I}-\frac{r}{2}\left(D_{I} \xi^{I}-C^{I} D_{I} s\right) \partial_{r}$

with $s(x)$ an arbitrary function on the 2-sphere.

Given the boundary conditions (3.2), the BMS charge (3.6) reduces to [7]

$$
\not \mathcal{Q}=\frac{1}{16 \pi G} \int_{S} d \Omega\left[\delta\left(-2 s F_{0}\right)+\frac{s}{2} \partial_{u} C_{I J} \delta C^{I J}\right] .
$$

The integrable part of the charge is given by

$$
\mathcal{Q}^{(\mathrm{int})}=-\frac{1}{8 \pi G} \int_{S} d \Omega s F_{0},
$$

while the nonintegrable part can be interpreted as the existence of Bondi flux at null infinity, which prevents the conservation of the charge along null infinity.

Alternatively, we may define the charge

$$
\mathcal{Q}=-\frac{1}{4 \pi G} \int d \Omega s\left(\psi_{2}^{0}+\sigma^{0} \partial_{u} \bar{\sigma}^{0}\right),
$$

where $\psi_{2}^{0}$ and $\sigma^{0}$ are defined in (1.2). As discussed in [5] [see Eq. (4.8) or (4.17) of Ref. [5]), one has

$$
\partial_{u} \mathcal{Q}=-\frac{1}{4 \pi G} \int d \Omega s\left(\left|\partial_{u} \sigma^{0}\right|^{2}-ð^{2}\left(\partial_{u} \bar{\sigma}^{0}\right)\right) .
$$

Newman and Penrose only considered the case where $s$ is taken to be an $\ell=0$ or $\ell=1$ spherical harmonic $Y_{\ell m}$, since after integration by parts on the second term one has a 
factor $\bar{\delta}^{2} Y_{\ell m}$, which vanishes identically. These $\ell=0$ and $\ell=1$ charges give the Bondi-Sachs mass and 3-momentum respectively [5]. In particular, the $\ell=0$ Bondi mass (or more precisely energy) is seen to be a strictly nonincreasing function of $u$, which is conserved if and only if $\partial_{u} \sigma^{0}=0$. In terms of the metric components defined in the expansions (3.2), one has

$$
\left|\partial_{u} \sigma^{0}\right|^{2}=\frac{1}{8} N^{I J} N_{I J},
$$

where $N_{I J}=\partial_{u} C_{I J}$ is the Bondi news tensor. Thus the Bondi-Sachs mass and 3-momentum are conserved if and only if the Bondi news tensor vanishes, signifying the absence of gravitational radiation at future null infinity $\mathscr{I}^{+}$.

More generally, we may allow the function $s$ in the charge (3.11) to be any arbitrary spherical harmonic, without the restriction to $\ell=0$ or $\ell=1$, and we again have charges that are conserved whenever the Bondi news tensor vanishes. ${ }^{4}$ Our focus in the remainder of this section will be on showing how these more general charges (3.11) are related to Barnich-Brandt BMS charges, and a generalization thereof.

Calculating $\psi_{2}^{0}$ and $\sigma^{0}$ in terms of the metric expansion coefficients in (3.2), one finds

$$
\psi_{2}^{0}+\sigma^{0} \partial_{u} \bar{\sigma}^{0}=\frac{1}{2} F_{0}-\frac{i}{4} D_{I} D_{J} \tilde{C}^{I J},
$$

and so the two expressions (3.10) and (3.11) are related by

$$
\mathcal{Q}^{\text {(int) }}=\Re(\mathcal{Q}),
$$

where we take $s$ to be an arbitrary function of $x^{I}$ in the definition of $Q$. This is analogous to what we found before in Sec. II, namely, for the asymptotic symmetry chosen to give a global charge, the BMS charge is the real part of the more general charge that we have defined in Eq. (3.11).

Noting that (3.15) has only provided a relation between the real part of the charge (3.11) and the Barnich-Brandt charge (3.10), and inspired by the electromagnetic example in the previous section, we are now led to consider the dual or magnetic Barnich-Brandt charge

$$
\not \tilde{\mathcal{Q}}=\frac{1}{8 \pi G} \lim _{r \rightarrow \infty} \int_{S} H=\frac{1}{8 \pi G} \lim _{r \rightarrow \infty} \int_{S} d \Omega \frac{H_{\theta \phi}}{\sin \theta}
$$

with $H$ defined in Eq. (3.7). It remains to show that this defines a charge, namely that the quantity defined above

\footnotetext{
${ }^{4}$ What one loses, by considering the infinity of charges corresponding to $\ell \geq 2$, is that now the nonconservation when $N_{I J} \neq 0$ is no longer of a definite sign, since both the $\partial^{2}\left(\partial_{u} \sigma^{0}\right)$ and the $\left|\partial_{u} \sigma^{0}\right|^{2}$ terms contribute when $N_{I J} \neq 0$. See, however, Appendix $\mathrm{C}$.
}

vanishes on-shell. We show that this is the case in Appendix A.

It is straightforward to show that (see Appendix B)

$\phi \tilde{\mathcal{Q}}=\frac{1}{16 \pi G} \int_{S} d \Omega\left[\delta\left(-s D_{I} D_{J} \tilde{C}^{I J}\right)+\frac{s}{2} \partial_{u} C_{I J} \delta \tilde{C}^{I J}\right]$,

where $^{5}$

$$
\tilde{C}^{I J}=C_{K}{ }^{(I} \epsilon^{J) K}, \quad \epsilon_{I J}=\left(\begin{array}{cc}
0 & 1 \\
-1 & 0
\end{array}\right) \sin \theta .
$$

Note that the nonintegrable term is closely analogous to that for $\not \mathcal{Q}$, see Eq. (3.9), and it also vanishes if the Bondi news vanishes. The integrable part gives rise to new charges

$$
\tilde{\mathcal{Q}}^{(\mathrm{int})}=-\frac{1}{16 \pi G} \int_{S} d \Omega s D_{I} D_{J} \tilde{C}^{I J}
$$

that are conserved in the absence of Bondi news. As can be seen from (3.14),

$$
D_{I} D_{J} \tilde{C}^{I J}=-4 \Im\left(\psi_{2}^{0}+\sigma^{0} \partial_{u} \bar{\sigma}^{0}\right),
$$

and so we have

$$
\mathcal{Q}=\mathcal{Q}^{(\text {int })}-i \tilde{\mathcal{Q}}^{(\mathrm{int})}
$$

Integrating by parts, $\tilde{\mathcal{Q}}^{\text {(int) }}$ in (3.19) can be rewritten as

$$
\tilde{\mathcal{Q}}^{(\mathrm{int})}=-\frac{1}{16 \pi G} \int_{S} d \Omega\left(D_{I} D_{J} s\right) \tilde{C}^{I J} .
$$

If $s$ is an $\ell=0$ or $\ell=1$ spherical harmonic, in which case $s$ satisfies $D_{I} D_{J} s=\frac{1}{2} \omega_{I J} \square s$, it follows that $\tilde{\mathcal{Q}}^{(\text {int })}=0$ since $\omega_{I J} \tilde{C}^{I J}=0$, and so one recovers the result [7] that $\mathcal{Q}=\mathcal{Q}^{\text {(int) }}$ for the $\ell=0$ and $\ell=1$ charges that correspond to the Bondi-Sachs 4-momentum.

In general, however, for an arbitrary function $s$ on the sphere, the $\tilde{\mathcal{Q}}^{\text {(int) }}$ are bona fide asymptotic charges in their own right, which supplement the already known BMS charges, $\mathcal{Q}^{\text {(int) }}$. Together, $\mathcal{Q}^{\text {(int) }}$ and $-\tilde{\mathcal{Q}}^{\text {(int) }}$ provide the real and imaginary parts of the generalized charges $\mathcal{Q}$ defined in (3.11).

\section{DISCUSSION}

We have shown that one can define new dual asymptotic charges at null infinity. These charges are the imaginary part of the charges defined in Eq. (3.11) - the real part

\footnotetext{
${ }^{5}$ In fact, $C_{K}{ }^{[I} \epsilon^{J] K}=0$, which can simply be shown using Schouten identities in two dimensions and the trace-free property of $C_{I J}$. Thus, $\tilde{C}^{I J}=C_{K}{ }^{I} \epsilon^{J K}$.
} 
being the charges of Barnich-Troessaert [7]. The new charges can be defined because at leading order it is possible to "dualize" the Barnich-Brandt 2-form to obtain an expression that also vanishes on-shell. In Ref. [2], it was shown that five of the ten conserved nonlinear NewmanPenrose charges are subleading charges in the BarnichBrandt formalism. It is, however, not possible to define dual Barnich-Brandt charges away from null infinity hence the question of how to fit the other five Newman-Penrose charges in the Barnich-Brandt formalism remains an open problem.

The existence of a further infinite number of BMS charges does not seem to give rise to new soft theorems $[12,13]$ as the imaginary part of $\psi_{2}^{0}$ at $\mathscr{I}_{ \pm}^{+}$and $\mathscr{I}_{ \pm}^{-}$is not part of the physical phase space [13]. However, we are nevertheless left with the question of the role of these charges in connection with the information paradox [14-16].

Dualising the Barnich-Brandt prescription only works for supertranslation charges and at null infinity. In particular, for the $\operatorname{SL}(2, \mathbb{C})$ part of the BMS group, the analysis of Appendix A does not go through, that is there are terms at order $r^{0}$ that are neither components of the Einstein equation nor total derivative terms; these terms provide an obstruction to a charge being defined. For the same reason, we cannot also understand the imaginary part of the extended BMS charges [2] in this way. It would, therefore, be helpful to understand why it was possible to define dual charges for supertranslations in terms of a more basic IyerWald [17] (see also Ref. [18]) or Barnich-Brandt [6] type of analysis.

\section{ACKNOWLEDGMENTS}

We would like to thank the Mitchell Family Foundation for hospitality at the Brinsop Court workshop where this work was initiated. M. G. is partially supported by Grant No. 615203 from the European Research Council under the FP7. C. N. P. is partially supported by DOE Grant No. DEFG02-13ER42020.

\section{APPENDIX A: BOUNDARY TERMS}

In this section, we prove that the variation of the dual charge (3.16) is equivalent to the Einstein equation. Starting from the definition of the charge, given in Eq. (3.16), and the fact that

$$
\delta g_{a b}=2 \nabla_{(a} \xi_{b)},
$$

a calculation similar to that done in Appendix D of Ref. [2] finds that

$$
\begin{aligned}
\not \tilde{\mathcal{Q}}= & \frac{1}{16 \pi G} \lim _{r \rightarrow \infty} \int_{S} d \theta d \phi \delta_{\theta \phi}^{I J}\left\{6 \xi_{J}\left[\nabla_{I}, \nabla_{c}\right] \xi^{c}\right. \\
& +2\left[\nabla_{J}, \nabla_{c}\right]\left(\xi_{I} \xi^{c}\right) \\
& \left.+2 \nabla_{c}\left(\xi_{I} \nabla^{c} \xi_{J}+\xi^{c} \nabla_{J} \xi_{I}-\xi_{I} \nabla_{J} \xi^{c}\right)\right\}
\end{aligned}
$$

Ignoring the first line in the expression above for now, the terms on the second line can be written as

$$
\frac{1}{8 \pi G} \lim _{r \rightarrow \infty} \int_{S} d \theta d \phi \delta_{\theta \phi}^{I J} \nabla_{c} X_{I J}{ }^{c},
$$

where

$$
X_{a b}^{c}=\xi_{a} \nabla^{c} \xi_{b}+\xi^{c} \nabla_{b} \xi_{a}-\xi_{a} \nabla_{b} \xi^{c} .
$$

Expanding out the integrand in Eq. (A3) gives

$$
\begin{aligned}
\nabla_{c} X_{I J}{ }^{c}= & \partial_{K} X_{I J}^{K}+\partial_{\hat{c}} X_{I J}^{\hat{c}}+\Gamma_{c e}^{c} X_{I J}^{e} \\
& -\Gamma_{d I}^{e} X_{[e J]}^{d}-\Gamma_{d J}^{e} X_{[I e]}^{d},
\end{aligned}
$$

where we use the notation that hatted lower case Latin indices, such as $\hat{c}$, denote $u$ or $r$ components. The first term is a boundary term and can, therefore, be ignored. Thus,

$$
\begin{aligned}
\nabla_{c} X_{I J}^{c}= & \partial_{\hat{c}}\left(g^{\hat{c} \hat{d}} X_{I J \hat{d}}\right)+\frac{1}{2} g^{-1} g^{\hat{c} d} \partial_{d} g X_{I J \hat{c}} \\
& -2 g^{\hat{c} d} \Gamma_{d I}^{K} X_{[K J] \hat{c}}-2 g^{c d} \Gamma_{d I}^{\hat{e}} X_{[\hat{e} J] c},
\end{aligned}
$$

where

$$
g \equiv \operatorname{det}\left(g_{a b}\right)=-r^{4} e^{4 \beta} \sin ^{2} \theta,
$$

and we have used the fact that the equation above is contracted with $\delta_{\theta \phi}^{I J}$, i.e., that the $I J$ indices are antisymmetrized and also the fact that

$$
X_{[I J] K}=X_{[I J K]}=0,
$$

which can be simply verified from the definition of $X_{a b c}$, Eq. (A4). Also, note that

$X_{[a b] c}=\xi_{[a} \partial_{|c|} \xi_{b]}-\xi_{c} \partial_{[a} \xi_{b]}-\xi_{[a} \partial_{b]} \xi_{c}=3 \xi_{[a} \partial_{c} \xi_{b]}$

since the Christoffel symbols cancel out. Moreover, as a direct consequence of the previous equation

$$
X_{[a b] c}=X_{[c a] b}=X_{[b c] a} .
$$

Consider the last term in Eq. (A6)

$-2 g^{c d} \Gamma_{d I}^{\hat{e}} X_{[\hat{e}]] c}=-2 g^{K d} \Gamma_{d I}^{\hat{e}} X_{[\hat{e} J] K}-2 g^{\hat{c} d} \Gamma_{d I}^{\hat{e}} X_{[\hat{e} J] \hat{c}}$.

First, we argue that the last term in the expansion above is an order $1 / r$ quantity as follows:

$$
\begin{aligned}
-2 g^{\hat{c} d} \Gamma_{d I}^{\hat{e}} X_{[\hat{e} J] \hat{c}} & =-2 g^{\hat{c} d} \Gamma_{d I}^{\hat{e}} X_{[\hat{c} \hat{e}] J} \\
& =-4 g^{d[u} \Gamma_{d I}^{r]} X_{[u r] J},
\end{aligned}
$$

where in the first equality above we used property (A10). Using the fact that 


$$
\begin{aligned}
& \xi_{u}=-\frac{1}{2}(\square s+2 s)+O(1 / r), \quad \xi_{r}=-s+O(1) \\
& \xi_{I}=-r \partial_{I} s+\frac{1}{2}\left(s D^{I} C_{I J}-D^{I} s C_{I J}\right)+O(1 / r),
\end{aligned}
$$

it is clear that

$$
X_{[u r] J}=O\left(r^{0}\right) .
$$

Moreover, using the expression for the Christoffel symbols given in section 4.3 of Ref. [19],

$$
-4 g^{d[u} \Gamma_{d I}^{r]}=2 e^{-4 \beta} g_{I L} \partial_{r} C^{L}=O(1 / r) .
$$

Hence, we find that

$$
\begin{aligned}
\delta_{\theta \phi}^{I J} \nabla_{c} X_{I J}{ }^{c}= & \partial_{\hat{c}}\left(g^{\hat{c} \hat{d}} X_{[\theta \phi] \hat{d}}\right)+\frac{1}{2} g^{-1} g^{\hat{c} d} \partial_{d} g X_{[\theta \phi] \hat{c}} \\
& +2 g^{\hat{c} d} \delta_{\theta \phi}^{I J} \Gamma_{d I}^{K} X_{[J K] \hat{c}} \\
& -2 g^{K d} \delta_{\theta \phi}^{I J} \Gamma_{d I}^{\hat{c}} X_{[J K] \hat{c}}+O(1 / r) .
\end{aligned}
$$

Note that

$$
\begin{aligned}
\delta_{\theta \phi}^{I J} X_{[J K] \hat{c}} & =2 \delta_{\theta \phi}^{I J} \delta_{J K}^{\theta \phi} X_{[\theta \phi] \hat{c}} \\
& =-\frac{1}{2} \delta_{K}^{I} X_{[\theta \phi] \hat{c}} .
\end{aligned}
$$

Thus,

$$
\begin{aligned}
\delta_{\theta \phi}^{I J} \nabla_{c} X_{I J}^{c}= & \partial_{\hat{c}}\left(g^{\hat{c} \hat{d}} X_{[\theta \phi] \hat{d}}\right) \\
& +\left[\frac{1}{2} g^{-1} g^{\hat{c} d} \partial_{d} g-g^{\hat{c} d} \Gamma_{d I}^{I}+g^{I d} \Gamma_{d I}^{\hat{c}}\right] X_{[\theta \phi] \hat{c}} \\
& +O(1 / r) .
\end{aligned}
$$

Now, consider the last two terms in the square brackets above

$$
\begin{aligned}
-g^{\hat{c} d} \Gamma_{d I}^{I}+g^{I d} \Gamma_{d I}^{\hat{c}}= & g^{\hat{c} e} g^{I d}\left(\partial_{d} g_{I e}-\partial_{e} g_{I d}\right) \\
= & g^{\hat{c} e} g^{I r} \partial_{r} g_{I e}+g^{\hat{c} K} g^{I J}\left(\partial_{J} g_{I K}-\partial_{K} g_{I J}\right) \\
& +g^{\hat{c} \hat{e}} g^{I J}\left(\partial_{J} g_{I \hat{e}}-\partial_{\hat{e}} g_{I J}\right) \\
= & g^{\hat{c} \hat{e}} g^{I J}\left(\partial_{J} g_{I \hat{e}}-\partial_{\hat{e}} g_{I J}\right)+O\left(1 / r^{2}\right) \\
= & g^{\hat{c} u} g^{I J} \partial_{J} g_{I u}-g^{\hat{c} \hat{e}} \frac{h^{I J}}{r^{2}} \partial_{\hat{e}}\left(r^{2} h_{I J}\right) \\
& +O\left(1 / r^{2}\right) \\
= & -\frac{4}{r} g^{\hat{c} \hat{e}} \partial_{\hat{e}} r+O\left(1 / r^{2}\right),
\end{aligned}
$$

where, in the last equality, we have used the fact that $h \equiv \operatorname{det}\left(h_{I J}\right)=\sin ^{2} \theta$. Using equations (A9) and (A13), one can show that

$$
\begin{aligned}
X_{[\theta \phi] u}= & -\frac{1}{2} r \delta_{\theta \phi}^{I J}\left\{\partial_{I} s\left[s D^{K} \partial_{u} C_{J K}-D^{K} s \partial_{u} C_{J K}\right]\right. \\
& \left.+\partial_{I} s \partial_{J} \square s\right\}+O(1),
\end{aligned}
$$

$X_{[\theta \phi] r}=\frac{1}{2} \delta_{\theta \phi}^{I J} s \partial_{I}\left[s D^{K} C_{J K}-D^{K}{ }_{s} C_{J K}\right]+O(1 / r)$,

which means the $O\left(1 / r^{2}\right)$ terms in Eq. (A19) can be consistently neglected. Thus,

$$
\begin{aligned}
\delta_{\theta \phi}^{I J} \nabla_{c} X_{I J}{ }^{c}= & \partial_{\hat{c}}\left(g^{\hat{c} \hat{d}} X_{[\theta \phi] \hat{d}}\right)+\left[\frac{1}{2} g^{-1} g^{\hat{c} d} \partial_{d} g-\frac{4}{r} g^{\hat{c} r}\right] X_{[\theta \phi] \hat{c}} \\
& +O(1 / r) .
\end{aligned}
$$

Now,

$\frac{1}{2} g^{-1} g^{\hat{c} d} \partial_{d} g=\frac{1}{2} g^{\hat{c} d} \frac{\partial_{d}\left(r^{4} e^{4 \beta} \sin ^{2} \theta\right)}{r^{4} e^{4 \beta} \sin ^{2} \theta}=\frac{2}{r} g^{\hat{c} r}+O\left(1 / r^{2}\right)$

and

$$
\begin{aligned}
\partial_{\hat{c}}\left(g^{\hat{c} \hat{d}} X_{[\theta \phi] \hat{d}}\right) & =\partial_{u}\left(g^{u r} X_{[\theta \phi] r}\right)+\partial_{r}\left(g^{u r} X_{[\theta \phi] u}\right)+O(1 / r) \\
& =-\partial_{u}\left(X_{[\theta \phi] r}\right)-\partial_{r}\left(X_{[\theta \phi] u}\right)+O(1 / r) \\
& =-\partial_{u}\left(X_{[\theta \phi] r}\right)-\frac{X_{[\theta \phi] u}}{r}+O(1 / r), \quad(\mathrm{A} 24)
\end{aligned}
$$

so that

$$
\delta_{\theta \phi}^{I J} \nabla_{c} X_{I J}^{c}=-\partial_{u}\left(X_{[\theta \phi] r}\right)+\frac{X_{[\theta \phi] u}}{r}+O(1 / r) .
$$

From equations (A20) and (A21)

$$
\begin{aligned}
\delta_{\theta \phi}^{I J} \nabla_{c} X_{I J}{ }^{c}= & -\frac{1}{2} \delta_{\theta \phi}^{I J} \partial_{I}\left\{s\left[s D^{K} \partial_{u} C_{J K}-D^{K} s \partial_{u} C_{J K}\right]\right. \\
& \left.+s \partial_{J} \square s\right\}+O(1 / r) .
\end{aligned}
$$

In summary, up to total derivative terms, which vanish upon integration

$$
\delta_{\theta \phi}^{I J} \nabla_{c} X_{I J}^{c}=O(1 / r)
$$

Going back to Eq. (A2) and using the fact that

$$
\begin{gathered}
{\left[\nabla_{a}, \nabla_{b}\right] V_{c}=R_{a b c}{ }^{d} V_{d},} \\
\not \tilde{\mathcal{Q}}=\frac{1}{4 \pi G} \lim _{r \rightarrow \infty} \int_{S} d \theta d \phi \xi_{[\theta} G_{\phi] c} \xi^{c},
\end{gathered}
$$

where $G_{a b}$ is the Einstein tensor. Hence $\not \tilde{\mathcal{Q}}$ vanishes onshell at leading order. 


\section{APPENDIX B: DERIVATION OF DUAL CHARGE}

In this appendix, we show that

$\lim _{r \rightarrow \infty} \frac{H_{\theta \phi}}{\sin \theta}=\frac{1}{2}\left\{\delta\left(-s D_{I} D_{J} \tilde{C}^{I J}\right)+\frac{s}{2} \partial_{u} C_{I J} \delta \tilde{C}^{I J}\right\}$,

where $H_{a b}$ is defined in Eq. (3.7). Note that

$$
\frac{H_{\theta \phi}}{\sin \theta}=\delta_{\theta \phi}^{I J} \frac{H_{I J}}{\sin \theta}=\frac{1}{2} \epsilon^{I J} H_{I J}
$$

Thus, from Eq. (3.7),

$$
\begin{aligned}
\frac{H_{\theta \phi}}{\sin \theta}= & \frac{1}{2} \epsilon^{I J}\left\{\xi_{J} g^{c d} \nabla_{I} \delta g_{c d}+\frac{1}{2} g^{c d} \delta g_{c d} \nabla_{J} \xi_{I}-\xi_{J} \nabla^{c} \delta g_{I c}\right. \\
& \left.+\xi^{c} \nabla_{J} \delta g_{I c}+\frac{1}{2} \delta g_{J c}\left(\nabla_{I} \xi^{c}-\nabla^{c} \xi_{I}\right)\right\} .
\end{aligned}
$$

Equations (3.8), with the falloff conditions (3.2), and (A13) give that

$$
\begin{array}{ll}
\xi^{u}=s, \quad \xi^{r}=\frac{1}{2} \square s+O(1 / r), & \xi^{I}=-\frac{1}{r} D^{I} s+O\left(1 / r^{2}\right), \\
\xi_{u}=-\frac{1}{2}(\square s+2 s)+O(1 / r), & \xi_{r}=-s+O\left(1 / r^{2}\right), \\
\xi_{I}=-r D_{I} s+O\left(r^{0}\right), &
\end{array}
$$

where $I, J, \ldots$ indices are lowered (raised) with the (inverse) metric on the round 2-sphere. Consider the first two terms in the expression on the right hand side of Eq. (B3). Using the expression for the determinant of metric given in Eq. (A7) and assuming implicitly the antisymmetrization in $[I J]$,

$$
\begin{aligned}
\xi_{J} g^{c d} \nabla_{I} \delta g_{c d}+\frac{1}{2} g^{c d} \delta g_{c d} \nabla_{J} \xi_{I} & =\xi_{J} \partial_{I}\left(g^{-1} \delta g\right)+\frac{1}{2} g^{-1} \delta g \partial_{J} \xi_{I} \\
& =4 \xi_{J} \partial_{I} \delta \beta+2 \delta \beta \partial_{J} \xi_{I} \\
& =O(1 / r)
\end{aligned}
$$

where we have used the falloff properties given in equations (3.2) and (B4). Hence, these terms will not contribute to the dual charge. Similarly, using Eqs. (3.2) and (B4) and the expression for the Christoffel symbols given in Sec. 4.3 of Ref. [19], it is fairly straightforward to show that

$$
\epsilon^{I J} \delta g_{J c}\left(\nabla_{I} \xi^{c}-\nabla^{c} \xi_{I}\right)=O(1 / r) .
$$

Hence,

$$
\frac{H_{\theta \phi}}{\sin \theta}=\frac{1}{2} \epsilon^{I J}\left\{\xi_{I} \nabla^{c} \delta g_{J c}+\xi^{c} \nabla_{J} \delta g_{I c}\right\}+O(1 / r) .
$$

Now, consider the first term above:

$$
\begin{aligned}
\epsilon^{I J} \xi_{I} \nabla^{c} \delta g_{J c}= & \epsilon^{I J} \xi_{I}\left(g^{u r} \nabla_{r} \delta g_{u J}+g^{K L} \nabla_{K} \delta g_{J L}\right)+O(1 / r) \\
= & \epsilon^{I J} \xi_{I}\left[g ^ { K L } \left(\partial_{K} \delta g_{J L}-2 \Gamma_{K(J}^{M} \delta g_{L) M}\right.\right. \\
& \left.\left.-2 \Gamma_{K(J}^{u} \delta g_{L) u}\right)-g^{u r} \Gamma_{r J}^{K} \delta g_{u K}\right]+O(1 / r) \\
= & \epsilon^{I J} D_{I} \delta \delta\left(D^{K} C_{J K}+2 C_{0 J}\right)+O(1 / r) .
\end{aligned}
$$

However, note that Eq. (3.5) then implies that, in fact,

$$
\epsilon^{I J} \xi_{I} \nabla^{c} \delta g_{J c}=O(1 / r) .
$$

Thus, as before, making use of Eqs. (3.2) and (B4) and the expression for the Christoffel symbols given in Sec. 4.3 of Ref. [19]

$$
\begin{aligned}
\frac{H_{\theta \phi}}{\sin \theta}= & \frac{1}{2} \epsilon^{I J} \xi^{c} \nabla_{J} \delta g_{I c}+O(1 / r) \\
= & \frac{1}{2} \epsilon^{I J}\left(\xi^{u} \nabla_{J} \delta g_{u I}+\xi^{K} \nabla_{J} \delta g_{I K}\right)+O(1 / r) \\
= & \frac{1}{2} \epsilon^{I J}\left(D_{I}\left(s \delta C_{0 J}\right)+D^{K}\left(s D_{I} \delta C_{J K}\right)\right. \\
& \left.-s D_{K} D_{I} \delta C_{J}{ }^{K}+\frac{1}{2} s \partial_{u} C_{I K} \delta C_{J}^{K}\right)+O(1 / r) \\
= & \frac{1}{2}\left(-s D_{I} D_{J} \delta \tilde{C}^{I J}+\frac{s}{2} \partial_{u} C_{I J} \delta \tilde{C}^{I J}\right)+O(1 / r),
\end{aligned}
$$

where in the last equality above we have neglected total derivative terms, which will integrate to zero and have used definition (3.18).

In conclusion, we find that

$$
\lim _{r \rightarrow \infty} \frac{H_{\theta \phi}}{\sin \theta}=\frac{1}{2}\left\{\delta\left(-s D_{I} D_{J} \tilde{C}^{I J}\right)+\frac{s}{2} \partial_{u} C_{I J} \delta \tilde{C}^{I J}\right\} .
$$

\section{APPENDIX C: ALTERNATIVE DEFINITION OF A REAL CHARGE}

From the perspective of the Newman-Penrose formalism, it would also make sense to define charges $\hat{Q}$ according to

$$
\hat{Q}=-\frac{1}{4 \pi G} \int d \Omega s\left[\psi_{2}^{0}+\sigma^{0} \partial_{u} \bar{\sigma}^{0}+ð^{2} \bar{\sigma}^{0}\right] .
$$

Then, from (3.12), one has

$$
\partial_{u} \hat{Q}=-\frac{1}{4 \pi G} \int d \Omega s\left|\partial_{u} \sigma^{0}\right|^{2},
$$

which holds for any choice of $s$ and demonstrates more clearly that the charges are conserved for vanishing Bondi news.

We may reexpress $\hat{Q}$ in terms of the metric expansion coefficients in (3.2). Noting first that $ð^{2} \bar{\sigma}^{0}=\frac{1}{4} D_{I} D_{J}\left(C^{I J}+\right.$ $\left.i \tilde{C}^{I J}\right)$, we find that 


$$
\hat{Q}=-\frac{1}{8 \pi G} \int d \Omega s\left(F_{0}+\frac{1}{2} D_{I} D_{J} C^{I J}\right) .
$$

Thus $\hat{Q}$ is in fact purely real, although it differs from the real part of $\mathcal{Q}$ defined in (3.11), which is given by $\mathcal{Q}^{\text {(int) }}$ in (3.10) [see Eq. (3.15)], by the addition of the second term.
[1] E. Conde and P. Mao, BMS supertranslations and not so soft gravitons, J. High Energy Phys. 05 (2017) 060.

[2] H. Godazgar, M. Godazgar, and C. N. Pope, Subleading BMS charges and fake news near null infinity, arXiv: 1809.09076.

[3] M. Campiglia and A. Laddha, Asymptotic charges in massless QED revisited: A view from spatial infinity, arXiv: 1810.04619.

[4] E. Conde and P. Mao, Remarks on asymptotic symmetries and the subleading soft photon theorem, Phys. Rev. D 95, 021701 (2017).

[5] E. T. Newman and R. Penrose, New conservation laws for zero rest-mass fields in asymptotically flat space-time, Proc. R. Soc. A 305, 175 (1968).

[6] G. Barnich and F. Brandt, Covariant theory of asymptotic symmetries, conservation laws and central charges, Nucl. Phys. B633, 3 (2002).

[7] G. Barnich and C. Troessaert, BMS charge algebra, J. High Energy Phys. 12 (2011) 105.

[8] S. Ramaswamy and A. Sen, Dual-mass in general relativity, J. Math. Phys. (N.Y.) 22, 2612 (1981).

[9] G. Bossard, H. Nicolai, and K. S. Stelle, Gravitational multiNUT solitons, Komar masses and charges, Gen. Relativ. Gravit. 41, 1367 (2009).

[10] H. Bondi, M. G. J. van der Burg, and A. W. K. Metzner, Gravitational waves in general relativity: 7. Waves from axisymmetric isolated systems, Proc. R. Soc. A 269, 21 (1962).

[11] R. K. Sachs, Gravitational waves in general relativity: 8. Waves in asymptotically flat space-times, Proc. R. Soc. A 270, 103 (1962).

[12] A. Strominger, On BMS invariance of gravitational scattering, J. High Energy Phys. 07 (2014) 152.

[13] T. He, V. Lysov, P. Mitra, and A. Strominger, BMS supertranslations and Weinbergs soft graviton theorem, J. High Energy Phys. 05 (2015) 151.

[14] S. W. Hawking, M. J. Perry, and A. Strominger, Soft Hair on Black Holes, Phys. Rev. Lett. 116, 231301 (2016).

[15] S. W. Hawking, M. J. Perry, and A. Strominger, Superrotation charge and supertranslation hair on black holes, J. High Energy Phys. 05 (2017) 161.

[16] S. Haco, S. W. Hawking, M. J. Perry, and A. Strominger, Black hole entropy and soft hair, J. High Energy Phys. 12 (2018) 098.

[17] V. Iyer and R. M. Wald, Some properties of Noether charge and a proposal for dynamical black hole entropy, Phys. Rev. D 50, 846 (1994).

[18] R. M. Wald and A. Zoupas, A general definition of "conserved quantities" in general relativity and other theories of gravity, Phys. Rev. D 61, 084027 (2000).

[19] G. Barnich and C. Troessaert, Aspects of the BMS/CFT correspondence, J. High Energy Phys. 05 (2010) 062. 